\title{
Mitglieder-Liste der Schweizeriscken Mikrobiologischen
}

\section{Gesellschaft}

Membres de la Société Suisse de Microbiologie

A. Ehrenmitglieder - Membres honoraires:

Karrer, P., Prof. Dr., Spyristeig 30, Zurich

Sobernheim, G., em. ord. Prof. Dr., Grindel·wald

Reh, Th., Prof. Dr. med., 34, chemin de Saussac, Troinex-Genève

B. Ordentliche Mitglieder - Membres ordinaires:

Acklin, O., Dr., Marchwartstraße 50, Zurich

Albertini, A. von, Prof. Dr. med., Histopathol. Institut der Universität, Zurich

Allemann, O., Dr. sc. techn., Hirschengraben 6, Bern

Armangué, M., Dr. med., 60, quai G.-Ador, Geneve

Badoux, V., Dr. ès sc, 21, av. de Rumines, Lausanne

Baer, J., Prof, Dr., Institut de Zoologie de ГUniversité, Neuchâtel

Barandun, S., Dr. med., Tiefenauspital, Bern

Bauer, Ed., Dr. ès sc, 1, avenue Jomini, Lausanne

Baumgartner, H., PD. Dr. med. vet., Lindenweg 6, Zollikofen (BE)

Bavaud, Ch., Dr. med., méd. cantonal, Inst. d'Hygiène, Geneve

Beer, K., Dr. med., Hygiene-Institut der Universität, Bern

Bein, M., Dr. ès sc, 35, chemin de Trabandan, Lausanne

Béraneck, J., Dr. ès sc, Boine 29, Neuchâtel

Berger, E., Prof. Dr. med., Pilgerstraße 31, Basel

Bernoulli, R., Dr. med., Rheinsprung 1, Basel

Bertschmann, M., Frl. Dr. phil. II, Fröschmattstraße 32, Bern

Betschard, M., Dr. chem., Lebensmittel-Untersuch.-Anstalt, Brunnen

Bezzegh, Thomas, Dipl. Biolog-Chemiker, Zurich 8, Höschgasse 2

Bielser, W., St.-Galler-Ring 84, Basel

Bloch, H., Prof. Dr. med., University of Pittsburgh, Pittsburgh, Pa., USA

Blöchliger, G., Dr. ès sc, Afico S. A., Corseaux s. Vevey

Blumer, S., Dr. phil., Schönenbergstraße 85, Wädenswil

Böhni, E., Frl. Dr., Clarahofweg 38, Basel

Bonifas, V., Dr., Department of Microbiology, St. Louis University, 1402, South

Grand Boulevard, St. Louis 4, Missouri, USA Bourgeois, Edm., Dr. med. vet., Morgartenstraße

17, Luzern

732 Mitglieder-Liste der Schweizerischen Mikrobiologischen Gesellschaft

Bovey, R., Dr. ès sci., rue de la Gare 35 bis, Nyon

Brandis, H., Prof. Dr., Hygienisches Institut, Universität Göttingen

Brodhage, H., Dr. med., Bramberghöhe 5, Luzern

Bruhin, H., Dr. phil. II, Äußere Baslerstraße 225, Riehen 
Bühlmann, X., Dr. med. vet., Fürfelderstraße 36, Ríehen/Basel

Burgisser, H., Dr. med. vet., 18, av. Montagibert, Lausanne

Bürki, F., PD. Dr. med. vet., Sandrainstraße 72, Bern

Calpini, P., Chef du Service d'Hygiène de ГEtat du Valais, Sion

Castelli, V., Dr. ès sc, Istituto Biochimico S. A., Lugano 3

Cathomas, S., Dr. med., Ilanz, Grb.

Chodat, F., Prof. Dr. ès sc, Institut de Botanique générale de $\Gamma$ Université, Geneve

Clerc, J.-F., Prof. Dr. med., 17, av. de la Gare, Neuchâtel

Cottet, P., Dr. med., Pérolles 1, Fribourg

Dedie, O., Dr. ès sc, La Combe, Rolle

Dommen, G., Dr. sc. techn., Monery, Rumilly (Hte. Savoie), France

Dorner, W., Dr. ès sc, Pierrefleur 10, Lausanne

Dostal, V., Dr. med., Hildegardstraße 7, Liebefeld-Bern

Dufour, L.-F., Lie ès sc, 29, Bd Georges-Favon, Geneve

Eckmann, A., Dr. chem., Neubrückstraße 61, Bern

Ehrsam, H. R., Dr. med. vet., Bombachhalde 26, Zurich 10/49

Ettlinger, L., Prof. Dr., Bakt. Institut der ETH, Zurich

Favarger, C, Prof. Dr., Institut de Botanique de $\Gamma$ Université, Neuchâtel

Ferrero, A., Dr. ing. chim., 1, av. de Miremont, Geneve

Fey, H., Prof. Dr. med. vet., Vet.-Bakt. Institut der Universität, Bern

Fiechter, A., Dr. sc. nat., Inst. für landw. Bakteriologie, ETH, Zurich 6,

Universitätsstraße 6 Fischer, H., Prof. Dr., Pharmakol. Institut der Universität, Zurich Fleury, C,

PD. Dr. méd et sc, Ecole de Médecine, Geneve Flüge, M., dipl. ing. agr., 34, av. Major Davel,

Vevey Fraschina, F., Dr. med., Medico cantonale, Via Coremmo 4a, Lugano 3 Frei, W., Prof., Höhestraße 68, Zollikon Fuchs, A., Dr., Grünegg, Konolfingen Fust, Bernh., Prof. Dr. med.,

Mittlere Straße 26, Basel Gander, R., Dr., sc. nat., Weedstraße 1030, Heerbrugg (SG) Gaschen, H. L., Dr. ès sc, 9, av. W.-Fraisse, Lausanne Gasser, R., Dr. rer. nat., Kluserstraße 33, Basel Gäumann, E., Prof. Dr., Institut für spez. Botanik, ETH, Zurich Gay, M., lie ès sc, Beim Letziturm 9, Basel Geiger-Huber, M., Prof. Dr., Botanische Anstalt, Basel Geigy, Rud., Prof. Dr., Schweiz. Tropeninstitut, Socinstraße 57, Basel Gelzer, J., Dr. med., Kinderspital, Zurich $7 / 32$

Gillert, K.-E., Dr. med., Prinz-Handjery-Straße 25, Berlin-Zehlendorf Glauser, H., Directeur, Saphal, Vevey Gräub, E., Dr. med. vet., Junkerngasse 41, Bern

Grumbach, A., Prof. Dr. med., Hygiene-Institut der Universität, Zurich Gsell, O., Prof. Dr. med., Maiengasse 56, Basel Gubler, H. U., Dr. phil. II, Hygiene-Institut der Universität, Bern

Guggenheim, P., Dr. med., Dermatologische Klinik, Kantonsspital, Zurich 6 Haag, E., Dr., 24, Av. Week-Reynold, Fribourg Hässig, Alfr., Dr. med., Elfenaustraße 8, Muri (Bern) Hallauer, C, Prof. Dr. med., Hygiene-Institut der Universität, Bern Hauser-Hauser, E., Frau Dr., Gossau (Zch.) Hein, H., Dr. med., Luisenstraße 22, Konstanz a. B.

Membres de la Société Suisse de Microbiologie

733

Herbert, A., Rev. Dr., 65, Watson Ave., East Orange, New Jersey, USA

Hess, E., Prof. Dr., Vet.-Bakt. Institut der Universität, Selnaustraße 36, Zurich

Hiller, Chr., Dr. med., Habsburgerstr. 5, München 13

Hirsch, J., Prof. Dr., Luzernerring 101, Basel

Huber, L., Frl., Schweiz. Serum- und Impfinstitut, Bern 
Hurni, H, Dr. phil. II, Elfenaustraße 57, Muri (BE)

Jaag, O., Prof. Dr., Eidg. Anst. f. Wasserversorgung und Gewässerschutz, Zurich

Jadassohn, W., Prof. Dr. med., 38, av. de Champel, Geneve

Kästli, P., Prof. Dr., Eidg. Milchwirtschaftl.-Bakt. Anstalt, Liebefeld-Bem

Kellenberger, Ed., Prof. Dr. ès sc, Institut de Physique, Geneve

Keller, H, Dr. med. vet., Veterinaria AG., Zurich

Kern, H., Prof. Dr. rer. nat., Institut für spez. Botanik, ETH, Zurich

Kikuth, W., Prof. Dr. med., Himmelgeisterstraße 224, Düsseldorf

Kilchsperger, Gladi, Dr. med. vet., Gartendörfli, Gontenbach (Zch.)

Klein, P., Dr. med., Mannesmann-Ufer 8, Düsseldorf

Klingler, K., PD. Dr. med. vet., Vet.-Bakt. und Parasitol. Institut, Bern

Knüsel, F., Dr. phil. nat., Aarburgerstraße 33, Zofmgen

Koenig, M., Dr. rer. nat., Kienbergstraße 8, Basel

Kocher, V., Dr., Weissensteinstraße 53, Bern

Kradolfer, F., Dr. med., Bruderholzallee 202, Basel

Krech, U., PD. Dr. med., Schlüsselackerweg 3, Hilterfmgen/BE

Kugler, Alf, Dr., Sandoz AG., Basel

Kurmann, J., Dr. ès sciences, Eidg. Milchwirtschaftliche Versuchsanstalt, Liebefeld (BE)

Kuslys, A., Dr. med. vet., Gossetstraße 20, Wabern (BE)

Lambelet, E., Dr. med., Bläsistraße 9, Zurich 10/49

Lang-Porchet, Mme B., 7, Avant-Poste, Lausanne

Lavillaureix, J., Dr. med., Institut d'Hygiène, 3, rue Kæberlé, Strasbourg

Lebek, G., Dr. med., Deutsche Forschungsanstalt f. Tuberkulose, Ludolph-Brauer-

Institut, München Leuenberger, M., Dr. méd. vét., Vétérinaire cantonal, Geneve Leupold, U.,

Prof. Dr. phil., Institut f. allgem. Botanik der Universität, Zurich Leuthardt, F., Prof. Dr., Burain

6, Rüschlikon Liebermeister, K., PD. Dr. med., Walpurgisstraße 11, München Lindenmann, J.,

Dr., Eidg. Gesundheitsamt, Bern Löffler, H, Dr. med., Hygiene-Institut, Universität Basel

LöfHer, W., Prof. Dr., Zürichbergstraße 44, Zurich 7 Lugon, J., Dr. ès sc, Laboratoire d'analyses médicales, Sion Luisier, M., ing. agr., 44, Ch. Pierrefleure, Lausanne Lüthi, H, Dr. phil., Eidg. Versuchsanstalt, Wädenswil Marti, H. R., Dr. med., Med. Poliklinik, Universität Basel Meier, R., Prof. Dr., CIBA-Aktiengesellschaft, Basel Metaxas, M. N., Dr. med., Blutspendezentrum Zurich des Schvveiz. Roten Kreuzes,

Hirschengraben 60, Zurich Mohler, H., Prof. Dr., Stadtchemiker, Oskar-Bider-Straße 10, Zurich Moosbrugger, G., Dr., Eidg. Vakzine-Institut, Basel Mooser, H., Prof. Dr. med., Hygiene-Institut der Universität, Zurich Müller, F., Dr. med., Marschalstraße 39, Düsseldorf Müller, H., Dr. med., Kurhaus Quisisana, Heiden (App.) Müller, Th., Dr. med., Gesundheitsamt des Kantons Basel-

Stadt, St.-Alban-Vorstadt 25,

Basel Muster, I., Mme Dr. ès sc, 9, route de Florissant, Geneve Naef-Roth, St., Frau Dr.,

Lindenstraße 41, Zurich Neipp, L, Dr. ès sc, Äußere Baselstraße 329, Riehen

734 Mitglieder-Liste der Schweizerischen Mikrobiologischen Gesellschaft

Neyroud, M., Dr., 1, Rond-Point de Plainpalais, Geneve

Nicole, Alfr., Dr. ès sc, 9, rue Cavour, Geneve

Novel, H.-E., PD. Dr. ès sc, Institut d'Hygiène, Geneve

Oswald-Bohm, W., Dr. med., Friedeckstraße 26, Zurich 11

Paccaud, M. F., Dr. med., 22, av. de Champel, Geneve

Peneveyre, M., 7, Bd Anatole France, Boulogne s. Seine, Paris 
Petrilli, F. L., Prof. Dr., Istituto d'lgiene, Genova

Petuely, Friedrich, Dr., Geidorfgürtel 50, Graz

Pfenninger, H., dipl. ing. agr. ETH., Elfenweg 15, Zurich 2/38

Plattner, P., Prof. Dr., F. Hoffmann-La Roche \& Co. AG., Basel

Poetschke, G., PD. Dr. med., Gautingerstraße 15, Stockdorf b. München

Pongratz, Edm., Dr. ès sc, Institut d'Hygiène, Geneve

Posternak, Y., Mme, 22, chemin Krieg, Geneve

Privat, E., Dr. ès sc, Labor, d'analyses médicales, 21, Bd des Philosophes, Geneve

Py, G., Lie ès sc. phys., 33, Fbg de l'Hôpital, Neuchâtel

Radvila, P., Dr. med. vet., Schweiz. Serum- und Impfinstitut, Bern

Ramseier, H., dipl. ing., Dept. of Microbiology, J. Hillis Miller Health Center, University

of Florida, Gainesville, USA Reber, H., PD. Dr. med., Kluserstraße 12, Basel

Regamey, R.-H., Prof. Dr. med., Institut d'Hygiène de $\Gamma$ Université de Geneve Reichstein, T.,

Prof. Dr., Organ.-Chem. Anstalt der Universität,

St.-Johanns-Ring 19, Basel Reusser, P., Dr., Emanuel-Büchel-Straße 4, Basel Richard, O., Dr.

sc. techn., Gevrey Chambertin, Côte d'Or, France Richie, R., Tierarzt, Fröschenweg 11,

Birsfelden (BL) Riniker, P., Dr. med., villa Alta, via Canovacce, Muralto-Locarno Ritter, P., Dr.

sc. techn., Milchwirtschaftliche Versuchsanstalt, Liebefeld (BE) Ritter, W., Dr. phil., Eidg.

Milchwirtsch.-Bakt. Anstalt, Liebefeld (BE) Rivkine, A., Frl., Dr., 7, rue Bellot, Geneve Roch,

R., Dr. med., 5, chemin du Vallon, Geneve Roggo, J., Dr. ès sc, Institut d'Hygiène, Fribourg

Roschka, W., Dr. med., Hygiene-Institut, Graz Rosenberg, W., Dr., Robapharm, St.-Alban-

Rheinweg 174, Basel Rosset, W., Dr. ès sc, 4, chemin de Chandieu, Lausanne Roth, W., PD. Dr. med., Basel, Postfach 27 Roulet, M. A., 52, rue Pierre-à-Mazel, Neuchâtel Sackmann, W., Dr. med. vet., Sieglinweg 10, Riehen Sansonnens, R., Dr. med., 7, chemin Monsoleil, Geneve Saurer, A., Frl. Dr. med., Wilfriedstraße 4, Zurich 7/32 Sauter-Serwetnik, L., Dr. med., c/o St. Lukes Hospital, New York, N.Y. Saxer, E., PD. Dr. med. vet., Weiherstraße 10, Gümligen Schär, M., Dr., Eidg. Gesundheitsamt, Bollwerk 27, Bern Schenk, Cécile, Frau Dr., Freiestraße 131, Zurich 7 Schlegel-Oprecht, Eva, Dr. phil. II, Andeer (GR)

Schmid, H., Prof. Dr., Chem. Institut der Universität, Rämistraße 76, Zurich Schmidt, G., Dr. med., Dr. Wander AG., Bern Schmidt, H., Prof. Dr. med., Seelhofenstraße 23, Wabern (BE) Schneider, P. A., Dr. med. vet., 2, av. Solange, Lausanne Schneiter, P., PD. Dr. med., 14, av. Tissot, Lausanne Schnitzer, A., Dr. med., Birmensdorferstraße 1, Zurich 4 Schoch, A., Prof. Dr. med., Zeitglockenlaube 2, Bern Schoch, H., Dr. phil. II, Bottmingerstraße 84, Binningen Scholer, H. J., Dr. med., Hirzbrunnenstraße 19, Basel

Membres de la Société Suisse de Microbiologie 735

Schopfer, W., Prof. Dr., Botanisches Institut der Universität, Bern

Schuler, W., Prof. Dr., Finkelerweg 18, Arlesheim

Schwander, L. A., ing. agr., 25, Buckingham Avenue, Craighall Park, Johannesburg,

Südafrika Schwarz-Speck, M, Frau Dr. sc. nat. ETH, Hedwigstraße 12, Zurich 7/32 Schweizer,

R., Dr. med. vet., Wiesenweg 12, St. Gallen Semenitz, E., Dr. med., Sterzingerstraße 8, Innsbruck

Sinden, J. W., Prof. Dr., Dept. of Botany, Pennsylvania State College, Pa., USA Somm, H., Dr., c/o ROCO/Conservenfabrik, Rorschach (SG) Somogyi, J. S., Dr., Nidelbadstraße 82, Rüschlikon Staehelin, H., Dr. med., Sandoz AG., Basel

Staub, H., Prof. Dr., Medizinische Universitätsklinik, Bürgerspital, Basel Staub, M., Dr. phil., Kantonschemiker, Fehrenstraße 15, Zurich Steck, W., Prof. Dr., Veterinärmedizinische Klinik, 
Bern Steinmann, J., PD. Dr. med., 9, rue de Candolle, Geneve Stoll, A., Prof. Dr., Sandoz AG., Basel

Stoll, Chr., Dr. rer. nat., Institut f. spez. Botanik, ETH, Zurich Storck, H., Prof. Dr. med., Mittelbergsteig 7, Zurich 7/44

Stundl, K., PD. Dr. phil., Inst. f. biochem. Technologie, Techn. Hochschule, Graz Stüssi, D. B., dipl. ing., Eidg. milchwirtsch. Versuchsanstalt, Liebefeld (BE) Suter, E., Dr. med., Head Depart, of Microbiology, J. Hillis Miller Health Center,

University of Florida, Gainesville, USA. Taterka, W., Dr. med., AG. vorm. B. Siegfried, Zofingen Taugwalder, R., Dr. ès sc, St.-Clara-Apotheke, Basel Terrier, Ch., Prof. Dr., Institut de Botanique de 1'Université, Neuchâtel Thomann, O., Dr., Carl-Spitteler-Straße 16, Zurich 53 Tomcsik, J., Prof. Dr., Hygiene-Institut der Universität, Basel Tuescher, J.-L., Dr. ès. sc, Les Laurelles, Leysin Tulasne, R., Prof. Dr., Institut de Biologie bactérienne, Strasbourg Turian, G., Dr. ès sc, Institut de Botanique générale de l'Université, Geneve Ungar, F., Dr. med., Mittlere Straße 137, Basel Vetter, H., Dr. med., Hohlgasse 57, Aarau Vischer, W. A., Dr. med., Burgweg 35, Basel

Viscontini, M., Prof. Dr., Chemisches Institut der Universität, Zurich Vogt, R., Dr., Feldrainstraße 48, Liebefeld-Bern Vonarburg, H., Dr. med. vet., Emngerstraße 109, Bern Wälchli, O., Dr., Oberstraße 286, St. Gallen

Wanner, H., Prof. Dr., Institut für allgem. Botanik der Universität, Zurich Wantz, M., Dr. ès sc, Schweiz. Serum- und Impfinstitut, Bern Weigle, J., Prof. Dr., California Institute of Technology, Pasadena, Calif., USA. Weyer, F., Prof. Dr. rer. nat., Tropen-Institut, Bernhard-Nocht-Straße 74, Hamburg 4 Wiesinger, D., Frl. Dr. phil., Rigistraße 56, Basel Wiesmann, E., PD. Dr. med., Iddastraße 39, St. Gallen Wikén, T., Prof. Dr., Institut für Mikrobiologie der Technischen Hochschule,

Delft (Niederlande) Wildhaber, M., Dr., 2, rue du Peyrou, Neuchâtel Wille, H., Dr. sc. techn., Eidg. Versuchsanstalt, Liebefeld-Bern Wuhrmann, K., Dr., Hofstraße 119, Zurich 7/44 Wulff, Herta, Dr. phil., Wabernstraße 90, Bern Wyler, R., Dr. med. vet., Oberer Batterieweg 17, Basel Wyttenbach, H., Dr., Neubadrain 78, Binningen Zbinden, Chr., Dr., Afico S. A., La Tour-dePeilz Zink, A. O., Dr. med., Ackermannstraße 21, Zurich

736 Mitglieder-Liste der Schweizerischen Mikrobiologischen Gesellschaft

Zinkernagel, R., Dr. phil. II, Sieglinweg 12, Riehen (BS)

Zogg, H., PD. Dr., Kirchbodenstraße 34, Thalwil (ZH)

Zoliikofer, Ed., Prof. Dr., Milchtechn. Institut, ETH, Eisgasse 8, Zurich 4

C. Kollektivmitglieder - Membres collectifs:

Afico S. A., La Tour-de-Peilz

Aktiengesellschaft vorm. B. Siegfried, Zofmgen

Bally Schuhfabrik AG., Schönenwerd

Bell AG., Basel

Berneralpen-Milchgesellschaft, Stalden-Konolfingen

Cellulose-Fabrik AG., Attisholz, Post Luterbach (Solothurn)

CIBA, Aktiengesellschaft, Basel

Galactina und Biomalz AG., Belp (Bern)

J. R. Geigy AG., Basel

Ed. Geistlich Söhne AG., Wolhusen (Luzern)

Haco-Gesellschaft AG., Gümligen

Hefefabriken AG., Olten 
Hero, Konserven AG., Lenzburg

F. Hoffmann-La Roche \& Co. AG., Basel

Intern. Verbandstoff-Fabrik Schaffhausen, Neuhausen

Maggi-Unternehmungen AG., Kemptthal

Sandoz AG., Basel

Schweiz. Ferment AG., Basel

Schweiz. Käseunion, Monbijoustraße 47, Bern

Schweiz. Serum- und Impfinstitut, Bern

Steril Catgut Gesellschaft, Bohny \& Hess AG., Neuhausen a. Rheinfall

Versuchsstation Schweiz. Brauereien, Steinhaldenstraße 45, Zurich

Dr. A. Wander AG., Bern

Wild Heerbrugg AG., Heerbrugg (St. Gallen)

Zahnärztliches Institut der Universität, Basel

Zyma SA., Nyon 